\title{
The Volunteers in Research programme: supporting COVID-19 research and improving medical training in parallel $\leqslant$ 洸
}

\author{
Authors: Tamsin McKinnon, ${ }^{\mathrm{A}}$ Alastair Watson, ${ }^{\mathrm{B}}$ Liam Richards, ${ }^{\mathrm{A}}$ Jackie Sears, ${ }^{\mathrm{C}}$ Matthew $]$ Brookes ${ }^{\mathrm{D}}$ and \\ Christopher A Green ${ }^{\mathrm{E}}$
}

\begin{abstract}
COVID-19 has had an unprecedented impact on society, global healthcare and daily life. The redeployment of research staff to patient-facing roles in the NHS left a depleted workforce to deliver critical urgent public health research (UPHR). We aimed to support UPHR studies and medical student training by developing and implementing a medical student Volunteers in Research programme. We further sought to gain insights about medical students' perceptions of this programme. We collected prospective data and conducted a retrospective survey as part of a service evaluation to assess the value of this clinical research experience to students, as well as motivators and barriers to taking part. The Volunteers in Research programme successfully supported UPHR studies during the COVID-19 pandemic. We generated important insights to help aid the wider implementation of this programme nationally to support essential research and medical student education.
\end{abstract}

KEYWORDS: volunteer network, medical education, COVID-19, service evaluation, clinical research

DOI: $10.7861 /$ clinmed.2020-1072

\section{Introduction}

COVID-19 has had an unprecedented impact on society, global healthcare and daily life. ${ }^{1,2}$ In response to the pandemic, research

Authors: ${ }^{\text {A }}$ medical student, Birmingham Medical School, Birmingham, UK; ${ }^{B}$ medical student, Birmingham Medical School, Birmingham, UK, research assistant and publication manager, University of Southampton Faculty of Medicine, Southampton, UK and NIHR Southampton Biomedical Research Centre,

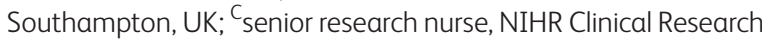
Network West Midlands, Wolverhampton, UK; ${ }^{\mathrm{D}}$ consultant clinical gastroenterologist, The Royal Wolverhampton NHS Trust, Wolverhampton, UK and Research Institute in Healthcare Science

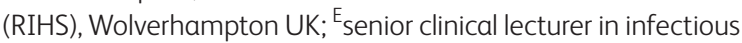
diseases, Institute of Microbiology and Infection, Birmingham, UK, consultant in infectious diseases and general medicine, University Hospitals Birmingham NHS Foundation Trust Birmingham, UK and NIHR Clinical Research Network West Midlands, Wolverhampton, UK and development staff were redeployed to patient-facing clinical roles in the NHS. ${ }^{3}$ This left a depleted workforce to deliver critical urgent public health research (UPHR) needed to characterise the new disease and identify potential treatments and vaccines. ${ }^{4}$

Within the West Midlands, the National Institute for Health Research (NIHR) Clinical Research Network (CRN) used this opportunity to fast-track the implementation of its novel Volunteers in Research programme. This programme aimed to recruit medical students to work alongside existing research staff to support UPHR studies. Medical students in the UK have reported limited opportunities to participate in research during medical school training, which may only arise if students choose to intercalate. ${ }^{5,6}$ Therefore, introduction of the Volunteers in Research programme had the additional aim of enabling medical students to experience clinical research first-hand and to develop skills essential for their careers as future doctors. ${ }^{7}$

Here, we describe medical student recruitment, training and deployment as part of the Volunteers in Research programme during the COVID-19 pandemic. Implementation of this programme helped support key UPHR including the International Severe Acute Respiratory and emerging Infections Consortium (ISARIC) study, RECOVERY trial and ChAdOx $1 \mathrm{nCoV}-19$ vaccine trial. ${ }^{8-11}$ To evaluate this programme, we collected prospective recruitment data and undertook a retrospective survey of students invited to take part to assess the perceived benefits, motivators and barriers to participating, as well as the skills students gained. This aimed to generate novel insights for medical education and aid the further development of this programme for wider national implementation.

\section{Methods}

\section{Volunteers in Research programme}

The Volunteers in Research programme began recruitment in midMarch 2020. An invitation was sent to regional medical schools, which was subsequently distributed to undergraduate students (Fig 1). Students expressed their interest in volunteering by contacting the email address provided. Students then completed an online course in good clinical practice (GCP) and provided their contact details, curriculum vitae and confirmation of a Disclosure and Barring Service check. The principal investigator briefed volunteers on their role at the NIHR before volunteers were assigned to different hospitals.

Students were allocated to their nearest NHS trust site based on their home postcode and formed small teams with supervision 


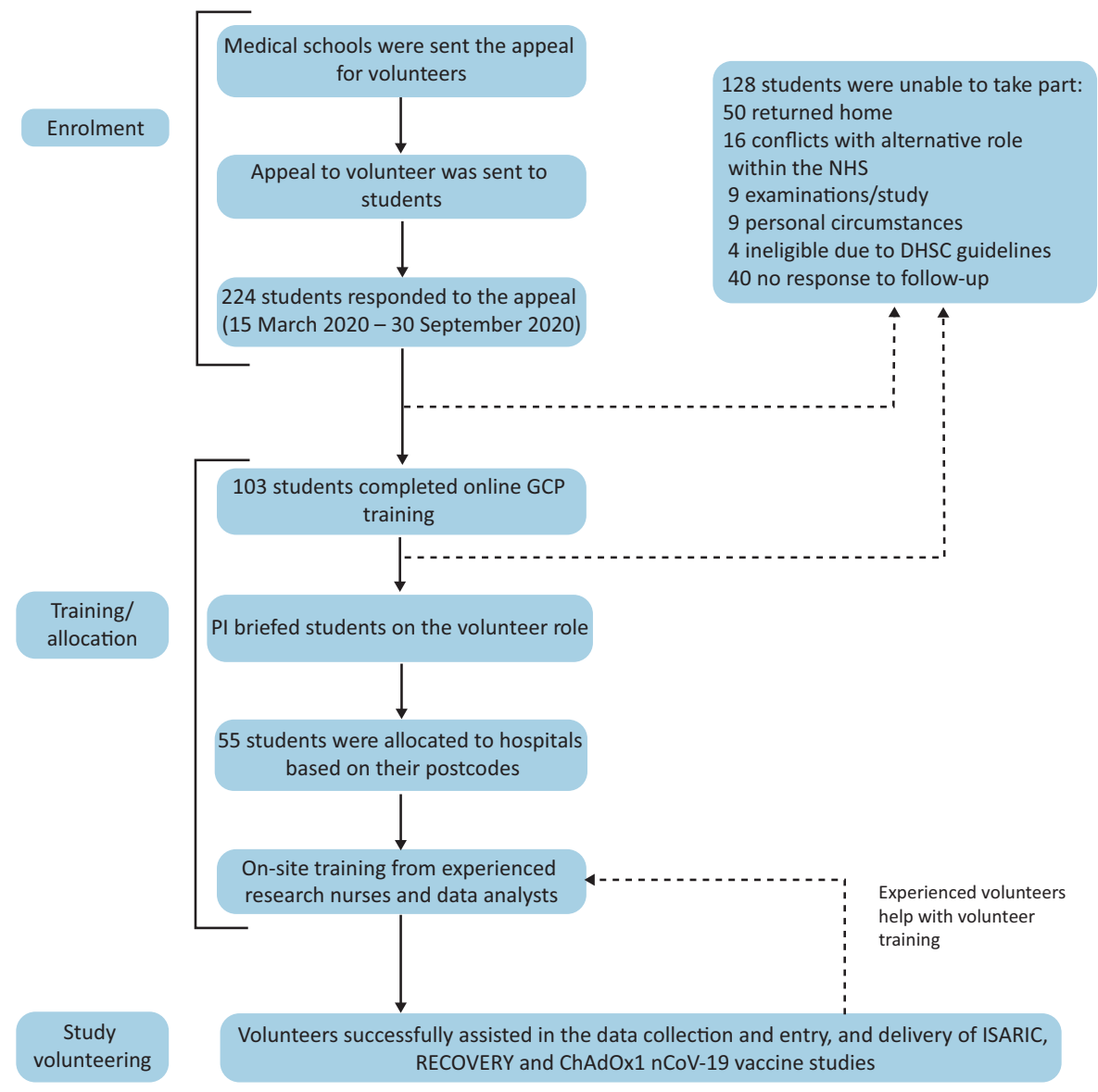

Fig 1. The recruitment and training pathway of medical student participants on the Volunteers in Research Programme. DHSC = Department of Health and Social Care; GCP = good clinical practice; $\mathrm{PI}=$ principle investigator. from a local team leader. Students received site-specific training from experienced research nurses, completed online training and read relevant trial protocols and other study documentation. Students were asked to cover a 'clinical trials assistant' level role, which involved tasks such as data collection, screening suitability of participants, sample processing and data entry as well as training of new students in their role. ${ }^{11}$ Prospective data was collected on volunteer recruitment and engagement, including a weekly survey asking students to log the number of hours worked.

\section{Service evaluation}

To evaluate this service, a survey was created using SurveyMonkey (SurveyMonkey, Palo Alto, USA) and distributed by email to all students who registered interest in the Volunteers in Research programme. The survey used branching logic to present students with different questions based on the extent of their involvement in the programme. The survey explored their perceptions of the benefits, motivators and barriers to taking part (supplementary material S1). We further explored participants' previous research experience and their opinion about further integration of research experience into the medical education curriculum. The survey was open from 15 July 2020 to 10 October 2020, during which time follow-up emails were sent. This was a service evaluation and as such no formal ethical approval was required.

\section{Data analysis and statistics}

Data was exported from SurveyMonkey and descriptive and summary statistics were calculated using GraphPad Prism v9.0.0.

\section{Results}

\section{Volunteers in Research programme}

From March to August 2020, 224 students responded to the appeal to take part in the Volunteers in Research programme. $46.0 \%(103 / 224)$ subsequently completed online GCP training (Fig 1), 53.4\% of whom (55/103) were then allocated to assist the delivery of UPHR studies at various hospital sites. Students who initially expressed interest but later reported being unable to take part in the programme gave a variety of reasons, including returning to their family home $(39.1 \%$; 50/128), conflicts with alternative roles $(12.5 \% ; 16 / 128)$, examinations or studying commitments $(7.0 \% ; 9 / 128)$, personal circumstances $(7.0 \% ; 9 / 128)$, being ineligible due to the UK Department of Health and Social Care guidelines $(3.1 \% ; 4 / 128)$ or no reason was given $(31.3 \%$; 40/128).

Students played key roles in the support and delivery of the ISARIC study, RECOVERY trial and ChAdOx1 $\mathrm{nCoV}-19$ vaccine trial. ${ }^{8-10}$ Students reported volunteering a total of 807.5 hours (33.6 hours/week) between March and August 2020, with the 
highest total of 524.5 hours being in May (131.1 hours/week; 2.4 hours/week/student).

\section{Volunteers in Research programme survey}

To gain insights into medical students' perceptions of the benefits, motivators and barriers to taking part, we distributed a survey to students who had registered interest in the Volunteers in Research programme. Of those who registered interest, $38.8 \%$ (87/224) responded to the survey. Using branching logic, students responded to questions tailored to the extent of their involvement in the programme, resulting in a variable total answered for each question (supplementary material S1). The mean age of respondents was 22.7 years (range 18-37) with $8.0 \%$ (7/87), $28.7 \%$ (25/87), $27.6 \%$ (24/87), $25.3 \%$ (22/87), and $10.3 \%(9 / 87)$ of the respondents being in academic years 1,2 , 3,4 and 5 , respectively. Of the respondents, $80.5 \%$ (70/87) were undergraduate students and $19.5 \%$ (17/87) were graduate-entry students. The majority $(58.6 \% ; 51 / 87)$ reported some form of prior research experience. Most respondents attended a medical school within the West Midlands, with 56.3\% (49/87) attending the University of Birmingham Medical School, 14.9\% (13/87) attending Keele University School of Medicine, 13.8\% (12/87) attending Aston Medical School, 6.9\% (6/87) attending Warwick Medical School; $8.1 \%$ (7/87) were from universities outside of the West Midlands (Table 1).

\section{Skill development}

Students reported developing various skills during the Volunteers in Research programme (Fig 2). 95.0\% (19/20) of participants reported that they had gained a new understanding of how clinical trials are conducted. The majority $(90.0 \% ; 18 / 20)$ reported improving their ability to interpret patient notes, with $80.0 \%(16 / 20)$ gaining a better understanding of clinical trial documentation. Students also reported gaining an understanding of COVID-19 disease and therapeutics (70.0\%; 14/20), an ability to implement multidisciplinary teamworking (70.0\%; $14 / 20)$, advanced communication skills $(65.0 \% ; 13 / 20)$, skills in data analysis and critical appraisal $(30.0 \% ; 6 / 20)$ and further understanding of informed consent $(30.0 \% ; 6 / 20)$.

\section{Motivators and barriers}

We sought to identify the motivators and barriers to taking part in the Volunteers in Research programme to aid the further development of this programme for wider implementation. The biggest motivators for students were 'Contributing to the NHS response to COVID-19', 'Making a difference during the crisis' and 'Getting hands-on experience of clinical research', with 90.3\% (56/62), 88.7\% (53/62) and 83.9\% (52/62) of the respondents reporting that this motivated them to take part 'A lot', respectively (Fig 3a). Other key motivators reported included 'Gaining a unique insight into the pandemic', 'The opportunity to network in the research community, 'Adding to my CV' and 'The opportunity to explore research as a career' with 69.4\% (43/62), 67.7\% (42/62), $46.8 \%(29 / 62)$ and $45.2 \%$ (28/62) of the respondents reporting that this motivated them to take part 'A lot', respectively.

Reported barriers to participating in the Volunteers in Research programme included 'Not having a way of getting to the site', 'Conflicts with other commitments or university studies', 'Moving
Table 1. Demographics of respondents to the Volunteers in Research programme survey

\begin{tabular}{|c|c|}
\hline Mean age, years & 22.7 \\
\hline Gender (male/female), n (\%) & $58(66.7)$ \\
\hline \multicolumn{2}{|l|}{ Ethnicity, n (\%) } \\
\hline White & $34(39.1)$ \\
\hline South-east Asian & $31(35.6)$ \\
\hline Chinese & $6(6.9)$ \\
\hline Black & $5(5.75)$ \\
\hline Other & $11(12.6)$ \\
\hline
\end{tabular}

Medical school, $\mathbf{n}(\%)$

University of Birmingham Medical $\quad 49(56.3)$

School

Keele University School of Medicine 13 (14.9)

Aston Medical School 12 (13.8)

Warwick Medical School 6 (6.9)

Other $\quad 7$ (8.1)

Undergraduate / graduate entry, n (\%) 70 (80.5) /

$17(19.5)$

Year of study, n (\%)

$\begin{array}{ll}\text { Year } 1 & 7(8.1) \\ \text { Year } 2 & 25(28.7) \\ \text { Year } 3 & 24(27.6) \\ \text { Year } 4 & 22(25.3) \\ \text { Year } 5 & 9(10.3)\end{array}$

Previous research experience, $\mathbf{n}(\%)$

Within degree (undergraduate/

postgraduate)

$23(32.9) /$

Previous employment

8 (11.4)

16 (22.9)

Intercalation year / elective

Audit or quality improvement

$8(11.4) / 0(0.0)$

$16(22.9)$

Observational or shadowing

$16(22.9)$

Other

$5(7.1)$

None

$19(27.1)$

Prior research type, $\mathrm{n}$ (\%)

Independent project

$31(47.0)$

Conference poster

$26(39.4)$

Lectures or seminars

$43(65.2)$

Workshops or small group teaching

$42(63.6)$

Other (please specify)

$1(1.5)$

home or away from the site', 'The risk of being exposed to COVID-19' and 'Lack of direction and instruction about the role' with $54.5 \%$ (36/66), $54.5 \%$ (36/66), 47.0\% (31/66), 45.5\% (30/66) and $40.9 \%(27 / 66)$ of the respondents, respectively, reporting that they found this to be a barrier 'A lot' or 'Slightly' (Fig 3b).

\section{Research opportunities in the medical curriculum}

We further aimed to understand whether medical students believed that the medical curriculum should include greater opportunities for students to participate in clinical research; $91.2 \%$ 


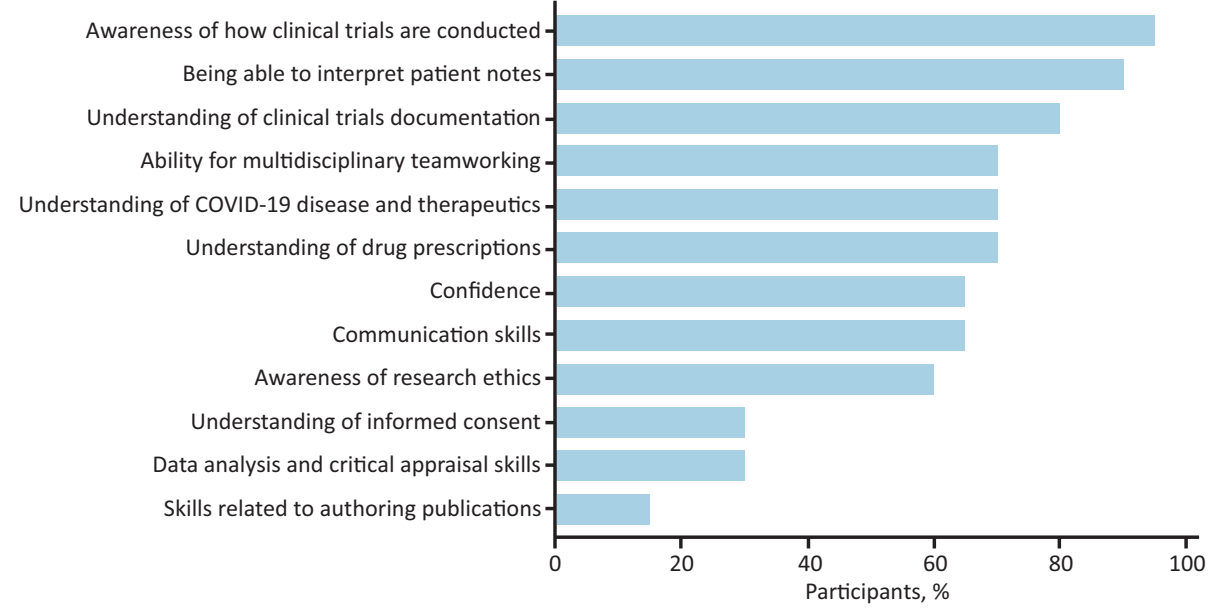

Fig 2. Medical student perceptions of skills gained from volunteering. Selected from a list the skills that they believed they had developed on the Volunteers in Research programme, $n=20$.
$(62 / 68)$ of respondents agreed that there should (Fig $4 a)$. When asked which research formats students would like to see included in the medical curriculum: $83.8 \%$ (57/68) reported wanting 'Independent research projects'; $64.7 \%$ (44/68), 'Shadowing opportunities'; 41.2\% (28/68), 'Lectures, seminars and workshops'; and $35.3 \%$ (24/68) wanting 'Online training' (Fig 4b). 73.5\%

$(50 / 68)$ of respondents reported that they would consider research as part of their future career.

\section{Discussion}

The Volunteers in Research programme successfully supported the delivery of UPHR studies during the COVID-19 pandemic and provided hands-on research experience for medical students. Our service evaluation identified the important motivators and barriers to taking part, insights that will aid the future development of this programme. We also highlight that students believe greater

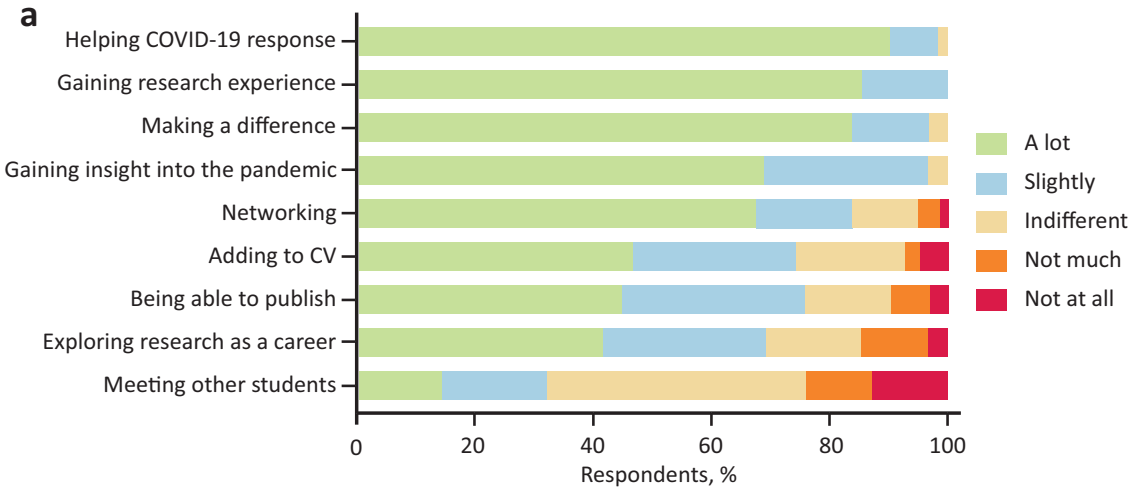

b

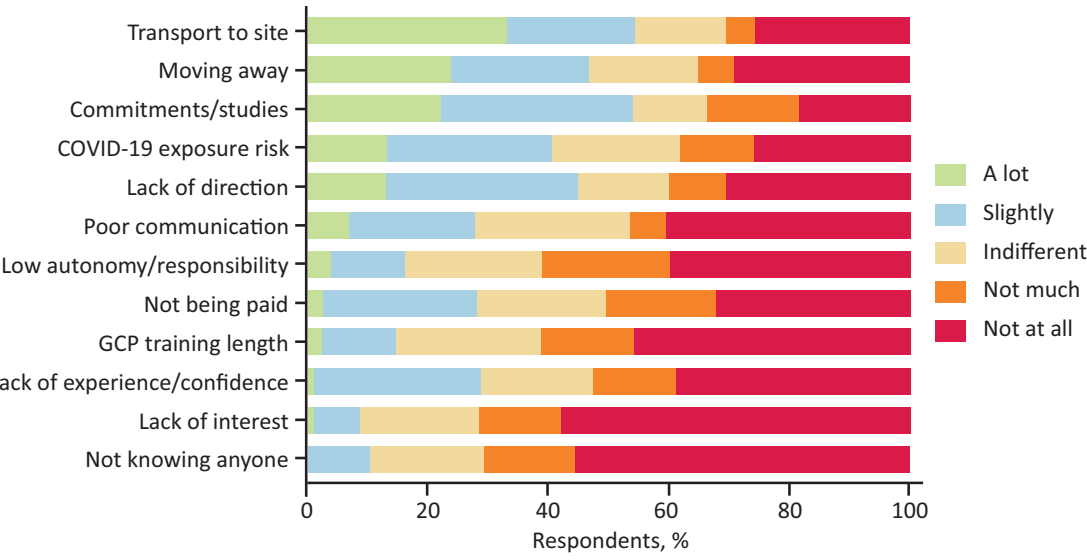

Fig 3. Motivators and barriers to taking part in the Volunteers in Research programme. a) Motivators, $n=62$. b) Barriers, $\mathrm{n}=65-67 . \mathrm{CV}=$ curriculum vitae; $\mathrm{GCP}=$ good clinical practice. 


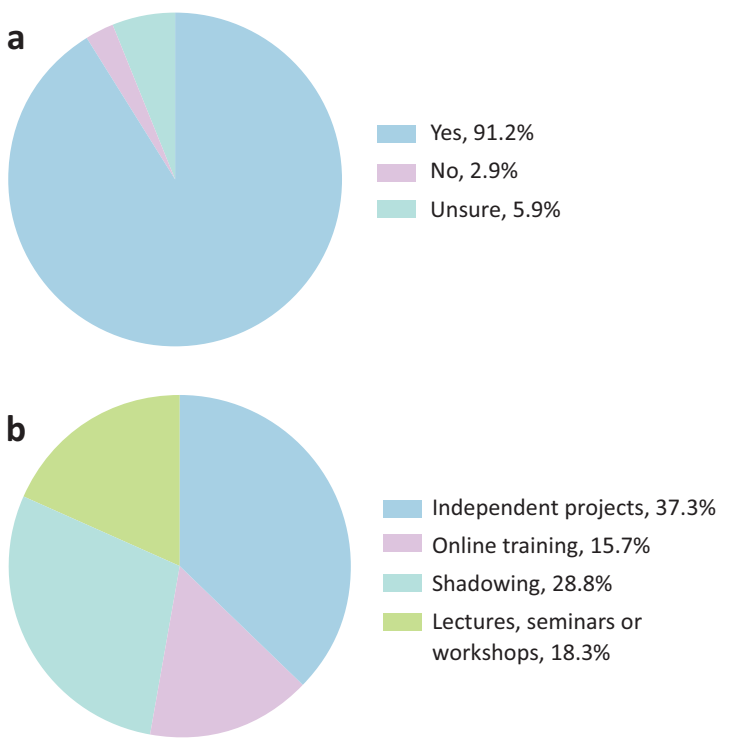

Fig 4. Medical student opinions about research experience in the medical curriculum. a) Should greater research opportunities should be included in the medical school curriculum, $n=68$. b) Which of the formats of research experience opportunities would they prefer, $n=68$.

opportunities to participate in research should be included in the medical school curriculum.

Through supporting UPHR studies including ISARIC, RECOVERY and ChAdOx1 nCoV-19, the Volunteers in Research programme facilitated the rapid characterisation of COVID-19 and identification of novel therapeutics and vaccines. ${ }^{8-10}$ Furthermore, the substantial medical student volunteering effort allowed the reallocation of experienced staff to patient facing clinical roles. Health services have faced numerous pressures during the COVID-19 pandemic, including staff working longer shifts and reporting higher levels of fatigue. ${ }^{12,13}$ Well-staffed rotas have been suggested to decrease stress and improve wellbeing among UK doctors during the pandemic, with staff redeployment playing a crucial role. $^{12,14}$

Medical student volunteers reported developing numerous skills, transferrable both to future research and clinical practice. Students improved their awareness of clinical trial conduct and documentation, data analysis skills and critical appraisal skills. This aligns with previous studies reporting the development of these skills during medical student research electives and research intercalation years. ${ }^{15,16}$ This programme could enable students to meet many of General Medical Council (GMC) outcomes for graduates, including the ability to critically appraise and apply medical research. ${ }^{17,18}$ In doing so, the Volunteers in Research programme may play a key role in enhancing the use of evidencebased medicine by future doctors. ${ }^{19}$

The number of full-time equivalent clinical academic staff employed by UK medical schools has been steadily decreasing. ${ }^{20}$ Furthermore, the recent decision to remove educational achievements from the total application score for entry onto the UK foundation programme in 2023 could negatively impact the motivation of students to seek out research opportunities, potentially leading to a further decline. ${ }^{21}$ Therefore, exposure to research through initiatives like this programme will be crucial in providing students with direct insights into clinical academic careers. Previous research has demonstrated the importance of early exposure to clinical trial settings, teaching on research methodology and elective research activities in promoting research pathways for medical students. ${ }^{22}$ However, medical students have reported limited opportunities to be involved in clinical research. $5,19,23$ Though most respondents in our service evaluation reported having prior research experience, this included experience of statistics tutorials, systematic review projects and lecture material. The vast majority of respondents believed that greater opportunities to get involved with clinical research should be made available within the medical school curriculum. The Volunteers in Research programme may, therefore, be a useful tool in providing these opportunities and promoting the future careers of clinical academics.

Medical students reported that key motivators for taking part in the programme included both supporting the NHS during the pandemic and gaining direct experience of clinical research, reflecting the dual aims of the programme. Even outside of a COVID-19 epidemic, the need for support for medical research will continue within an NHS that is increasingly resource limited. ${ }^{24}$ Previous studies have reported medical student motivations to taking part in research to include publications, presentation of findings, understanding research methodology, developing critical appraisal skills and pursuing a career in research, motivations which agreed with those found within this Volunteers in Research programme service evaluation. ${ }^{25}$ Studies have also highlighted the importance of prior experience, an understanding of what research entails and confidence in encouraging medical student participation in clinical research. ${ }^{15,22}$ Engaging students through the Volunteers in Research programme and communicating the support within this role will be key for students taking part in this programme and may give them confidence and experience to take part in future research.

This programme was fast-tracked by the simultaneous release of medical students from their studies and the redeployment of clinical researchers to ward-based roles. However, there is potential to implement this programme more widely to support both COVID-19 and non-COVID-19 research studies and compliment medical student training and objectives set out in other parts of the curriculum. Nevertheless, there are a number of hurdles to overcome. In comparison with motivators, the barriers that medical students reported were more varied, with few students reporting that any single barrier influenced their decision 'A lot'. Moving back home for lockdown was cited as a barrier to participating. Though this will be less relevant outside of a COVID-19 lockdown, it could be a consideration in between academic terms. Similarly, the requirement for student transport to research sites should be reflected on. The need for high levels of supervision in clinical research could limit how many clinical research places will be made available to students. However, this and the lack of direction about the role could be overcome by formally incorporating the programme into the curriculum, and ensuring effective instruction, support, training and communication; this will likely be less challenging outside of the unprecedented environment of a COVID-19 epidemic. Other barriers to engagement will inevitably persist, including conflicts with other commitments, especially when trying to implement this programme as part of an already busy medical education curriculum. 
A significant number of total hours were volunteered during the programme and, due to the low response rate of weekly working timesheet questionnaires, this is likely an underestimate. However, over the whole period, only a relatively small number of hours were worked on average each week per student, which was sufficient to support the research studies and medical student education in parallel. It seems feasible, therefore, that spending relatively little time embedded within clinical research teams would be enough to confer many of the benefits found within the service evaluation.

The Volunteers in Research programme successfully recruited a motivated, self-selected group of students from all academic years who demonstrated commitment to delivering high quality research objectives. This reflects the self-selected group of doctors who choose to pursue a career in clinical academia. However, this group may not be representative of the wider medical student cohort, which is an important consideration when reflecting on the generalisability of this approach. A disproportionate number of graduate-entry medical students volunteered within this programme, reflecting the reported increased frequency of graduate-entry students undertaking research roles in their future careers. ${ }^{26}$ Further research to characterise the opinions of a non-self-selected group of medical students would be beneficial. A proportion of students may not want to engage in such a programme, and voluntary participation may therefore be the best approach.

Our service evaluation, like all service evaluations, has its limitations. Our survey used preselected statements for participants to consider. These were chosen through development with a focus group of students and an additional text box was provided for students to provide further opinions. However, further evaluation could use a wider qualitative approach to uncover additional insights. The survey was sent to all participants who showed an interest in the programme and only $36.1 \%$ of participants responded. However, this is a similar response rate to that of other service evaluations. ${ }^{27}$

\section{Conclusion}

We have successfully implemented a medical student Volunteers in Research programme to support clinical research during the COVID-19 pandemic. This helped with the rapid characterisation of COVID-19 hospitalisations and the development of novel therapeutics and vaccines. Students who took part gained numerous transferrable skills and found the experience helped to inform future career options. Through performing a service evaluation, we have gained insights into the benefits of this programme and motivators and barriers for students taking part, which can be used to support wider implementation of similar programmes and improve medical education.

\section{Supplementary material}

Additional supplementary material may be found in the online version of this article at www.rcpjournals.org/clinmedicine: S1 - Questionnaire for Volunteers in Research service evaluation.

\section{Acknowledgements}

We would like to thank all participants of the Volunteers in Research programme and the NIHR CRN West Midlands COVID-19 medical student volunteers administrative team for their support in helping to set up and undertake this work, particularly Debbie Peplow and Jackie Sears for their dedicated help to students.

\section{Conflicts of interest}

Matthew ] Brookes has received grants and travel expenses from Vifor International and Tillots Pharma outside of the submitted work.

\section{References}

1 Watson A, Wilkinson TM. Respiratory viral infections in the elderly. Ther Adv Respir Dis 2021;15:1753466621995050.

2 World Health Organization. Coronavirus disease (COVID-19): Situation report -103. WHO, 2020. www.who.int/docs/defaultsource/coronaviruse/situation-reports/20200502-covid-19sitrep-103.pdf?sfvrsn=d95e76d8_4 [Accessed 02 May 2020].

3 Vindrola-Padros C, Andrews L, Dowrick A et al. Perceptions and experiences of healthcare workers during the COVID-19 pandemic in the UK. BMJ Open 2020;10:e040503.

4 Thornton J. Clinical trials suspended in UK to prioritise covid-19 studies and free up staff. BMJ 2020;368:m1172.

5 Metcalfe D. Involving medical students in research. J $R$ Soc Med 2008;101:102-3.

6 Griffin MF, Hindocha S. Publication practices of medical students at British medical schools: experience, attitudes and barriers to publish. Med Teach 2011;33:e1-8.

7 Watson A, McKinnon T, Prior S-D, Richards L, Green CA. COVID-19: time for a bold new strategy for medical education. Medical Education Online 2020;25:1764741.

8 Docherty AB, Harrison EM, Green CA et al. Features of 20133 UK patients in hospital with covid-19 using the ISARIC WHO Clinical Characterisation Protocol: prospective observational cohort study. BMJ 2020;369:m1985.

9 RECOVERY Collaborative Group. Dexamethasone in hospitalized patients with Covid-19_-preliminary report. N Engl ] Med 2020;384:693-704.

10 Voysey M, Clemens SAC, Madhi SA et al. Safety and efficacy of the ChAdOx1 nCoV-19 vaccine (AZD1222) against SARS-CoV-2: an interim analysis of four randomised controlled trials in Brazil, South Africa, and the UK. Lancet 2021;397:99-111.

11 Prior S-D, McKinnon T, Gresty V et al. COVID-19: medical students in clinical research. Clin Teach 2021;18:79-81.

12 Faderani R, Monks M, Peprah D et al. Improving wellbeing among UK doctors redeployed during the COVID-19 pandemic. FHJ 2020;7:e71-6.

13 Willan J, King AJ, Jeffery K, Bienz N. Challenges for NHS hospitals during covid-19 epidemic. BMJ 2020;368:m1117.

14 Tanne JH, Hayasaki E, Zastrow M et al. Covid-19: how doctors and healthcare systems are tackling coronavirus worldwide. BMJ 2020;368:m1090.

15 Murdoch-Eaton D, Drewery S, Elton S et al. What do medical students understand by research and research skills? Identifying research opportunities within undergraduate projects. Med Teach 2010;32:e152-60.

16 Houlden RL, Raja JB, Collier CP, Clark AF, Waugh JM. Medical students' perceptions of an undergraduate research elective. Med Teach 2004:26:659-61.

17 General Medical Council. Outcomes for graduates. GMC, 2020. www.gmc-uk.org/education/standards-guidance-and-curricula/ standards-and-outcomes/outcomes-for-graduates/outcomes-forgraduates/outcomes-3-professional-knowledge\#clinical-researchand-scholarship [Accessed 21 November 2020].

18 Buckland R. Medical student volunteering during COVID-19: lessons for future interprofessional practice. J Interprof Care 2020;34: 679-81.

19 Meats E, Heneghan C, Crilly M, Glasziou P. Evidence-based medicine teaching in UK medical schools. Med Teach 2009;31:332-7.

20 Rimmer A. Clinical academic workforce continues to shrink. BMJ 2017;358:j3352. 
21 Nabavi N. BMA strongly opposes changes to foundation programme applications. BMJ 2020;371:m4837.

22 Rosenkranz SK, Wang S, Hu W. Motivating medical students to do research: a mixed methods study using Self-Determination Theory. BMC Med Educ 2015;15:95.

23 Taylor AK, Purdy S. How to get involved in research as a medical student. BMJ 2017:356:16593.

24 Appleby J, Galea A, Murray R. The NHS productivity challenge. Experience from the front line. London: The King's Fund. 2014.

25 Oliveira CC, de Souza RC, Abe ÉHS et al. Undergraduate research in medical education: a descriptive study of students' views. BMC Med Educ 2014;14:51.
26 Rolfe IE, Ringland C, Pearson S-A. Graduate entry to medical school? Testing some assumptions. Med Educ 2004;38:778-86.

27 Baruch Y, Holtom BC. Survey response rate levels and trends in organizational research. Human Relations 2008;61:1139-60.

Address for correspondence: Dr Christopher A Green, Institute of Microbiology and Infection, University of Birmingham, Birmingham B15 2TT, UK.

Email: c.a.green.2@bham.ac.uk 